\title{
SUR UN THÉOREME DE M. FUCHS
}

PAR

\section{H. POINCARÉ}

a $P$ A R IS.

Les équations différentielles linéaires jouissent d'une propriété remarquable: Les points singuliers sont les mêmes pour toutes les intégrales. C'est ainsi que pour les équations dont les coëfficients sont des polynòmes entiers en $x$, les points singuliers sont les valeurs de $x$ qui annulent le premier coëfficient. C'est sur cette circonstance qu'est fondée la méthode d'intégration de ces équations par les fonctions zétafuchsiennes.

Les équations non linéaires ne jouissent pas, du moins en général, de la même propriété. Ainsi l'équation très simple

$$
x d x+y d y=0
$$

a pour intégrale générale:

$$
y=\sqrt{c^{2}-x^{2}}
$$

$c$ étant une constante d'intégration. Et les points singuliers $x= \pm c$, dépendent de cette constante et ne sont par conséquent pas les mêmes pour toutes les intégrales.

On est ainsi conduit à rechercher s'il existe, en dehors des équations linéaires, d'autres classes d'équations différentielles dont toutes les intégrales particulières aient les mêmes points singuliers. C'est ce problème que M. Fuchs a très élégamment résolu dans un mémoire intitulée Ueber Differentialgleichungen deren Integrale feste Verzweigungspunkte be- 
sitzen et inséré aux Sitzungsberichte de l'Académie de Berlin. (Séance du 26 Juin i 884.)

Je rappelle succinctement les notations employées par le savant géomètre de Berlin et les résultats qu'il a obtenus.

M. Fuchs considère une équation du $\mathrm{I}^{\text {er }}$ ordre:

$$
F\left(z \cdot y, y^{\prime}\right)=0
$$

où $z$ est la variable indépendante et $y^{\prime}$ la dérivé $\frac{d y}{d z}$ et dont le premier membre est une polynòme entier en $y$ et en $y^{\prime}$, ayant pour coëfficients des fonctions quelconques de $z$.

Si l'on considère un instant $z$ comme une constante, l'équation (A) devient une relation algébrique entre $y$ et $y^{\prime}$. On appelle $p$ le genre de cette relation.

\section{L'équation}

$$
D(z, y)=\mathrm{o}
$$

est celle que l'on obtient en éliminant $y^{\prime}$ entre l'équation (A) et la suivante:

$$
\frac{\partial F}{\partial y^{\prime}}=0 \text {. }
$$

M. Fuchs arrive d'abord à un résultat général qu'il énonce ainsi:

Die nothwendigen und hinreichenden Bedingungen dafür, dass die Integrale der Gleichung (A) feste, sich nicht mit den Änderungen der Anfangswerthe stetig verschiebende Verzweigungspunkte besitzen, sind die folgenden:

I. Die Gleichung (A) hat die Form:

$$
y^{\prime m}+\psi_{1} y^{\prime m-1}+\phi_{2} y^{\prime m-2}+\ldots+\psi_{m}=0
$$

worin $\psi_{1}, \psi_{2}, \ldots, \psi_{m}$ ganze rationale Functionen von $y$ mit von $z$ abhängigen Coefficienten von der Beschaffenheit bedeuten, dass $\varphi_{k}^{\prime}$ höchstens vom Grade $2 k$ in Bezug auf $y$ ist.

2. Ist $y=\eta$ eine Wurzel der Discriminantengleichung (C) für welche die durch (F) definirte algebraische Function $y^{\prime}$ von $y$ sich verzweigt so ist $\eta$ ein Integral der Gleichung (F). In der $y^{\prime}$ als 
algebraische Function von $y$ darstellenden Rismavs'schen Fläche hat $y^{\prime}$ in sämmtlichen über $y=\eta$ liegenden Verzweigungsstellen den Werth $y^{\prime}=\zeta=\frac{d \eta}{d z}$.

3. Je a Blättern, welche sich in $y=\eta, y^{\prime}=\xi=\frac{d \eta}{d z}$ verzweigen, entsprechen mindestens $x$ - I mit $y=y$ zusammenfallende Wurzeln der Gleichung

$$
F(z, y, \xi)=0
$$

mit der Unbekannten $y$.

En d'autres termes; l'équation (A) devra satisfaire aux conditions suivantes:

$I^{\circ}$. La fonction $y^{\prime}$ définie par cette équation ne pourra devenir infinie que lorsque $y$ sera lui-même infini, ou pour certaines valeurs particulières de $z$.

$2^{\circ}$. Si l'on pose $y_{1}=\frac{1}{y}, y_{1}^{\prime}=\frac{d y_{1}}{d z}$, l'équation (A) deviendra:

$$
F_{1}\left(z, y_{1}, y_{1}^{\prime}\right)=0
$$

$y_{1}^{\prime}$ ne devra pouvoir devenir infinie, si $y_{1}$ est nul, que pour certaines valeurs particulières de $z$.

$3^{\circ}$. Les équations:

$$
F=\frac{\partial F}{\partial y^{\prime}}=\mathrm{o}
$$

devront définir des intégrales singulières de l'équation (A).

$4^{\circ}$. En différentiant l'équation (A), on trouve:

$$
\frac{d F^{\prime}}{d y^{\prime}} \frac{d y^{\prime}}{d z}+\frac{d F^{\prime}}{d y} y^{\prime}+\frac{d F^{\prime}}{d z}=\text { o. }
$$

On devra avoir identiquement:

$$
\frac{d F}{d y} y^{\prime}+\frac{d F^{\prime}}{d z}=P F+Q \frac{d F^{\prime}}{d y^{\prime}}
$$

$P$ et $Q$ étant des polynòmes entiers en $y$ et en $y^{\prime}$, ayant pour coëfficients des fonctions de $z$.

Il est aisé de comprendre l'importance de ces résultats. Supposons en effet que $F$ soit un polynôme entier, non seulement par rapport à $y$ 
H. Poincaré.

et à $y^{\prime}$, mais encore par rapport à $z$. Alors, si les conditions précédemment énoncées sont remplies, le nombre des points singuliers est fini et ces points peuvent même être regardés comme donnés, de sorte que la méthode d'intégration des équations linéaires par les fonctions fuchsiennes est applicable, au moins dans ses traits essentiels. On pourrait donc ainsi concevoir l'espoir de découvrir une classe nouvelle d'équations différentielles intégrables par ces transcendantes. Dans le cas mème où $F$ n'est pas un polynôme entier par rapport à $z$, le résultat reste fort important.

Mais pour en tirer tous les fruits, il est indispensable de faire des conditions précédemment énoncées unè étude plus approfondie. Cette étude a été commencée et poussée assez loin par M. Fuchs et je désirerais ici la pousser plus loin encore, afin d'arriver à des conclusions définitives.

Le nombre que nous avons appelé plus haut $p$ joue dans cette étude un rôle capital.

I $^{\circ}$. Supposant d'abord $p=0$, M. Fuchs pose

$$
y=\frac{\Phi_{1}(t)}{\Phi_{0}(t)}, \quad y^{\prime}=\frac{\Phi_{2}(t)}{\Phi_{0}(t)}
$$

$\Phi_{0}, \Phi_{1}$ et $\Phi_{2}$ désignant des polynômes entiers en $t$, dont les coëfficients dépendent de $z$. Il arrive ainsi à l'équation

$$
\frac{d t}{d z}=A_{0}+A_{1} t+A_{2} t^{2}
$$

où $A_{0}, A_{1}, A_{2}$ sont des fonctions de $z$. C'est l'équation de Riccati qu'il est aisé de ramener, comme on sait, aux équations linéaires du $2^{\mathrm{d}}$ ordre. Ainsi dans le cas de $p=0$, on n'obtient pas de classe réellement nouvelle d'équations différentielles satisfaisant aux conditions énoncées.

Dans ce premier cas, je n'ai rien à ajouter aux résultats obtenus par M. Fuchs.

Ce savant géomètre, examinant ensuite le cas de $p=\mathrm{I}$, pose:

$$
y=\frac{\Phi_{1}+\Psi_{1} \sqrt{R(t)}}{\Phi_{0}+\Psi_{0} \sqrt{R(t)}}, \quad y^{\prime}=\frac{\Phi_{2}+\Psi_{2} \sqrt{R(t)}}{\Phi_{0}+\Psi_{0} \sqrt{R(t)}}
$$

les $\Phi$, les $\psi$ et $R$ étant des polynômes entiers en $t$ avec des coëfficients dépendant de $z$, et $R$ en particulier étant du $4^{\mathrm{e}}$ degré en $t$. 
L'équation (A) est alors ramenée ì la forme:

$$
\frac{d t}{d z}=A_{0}+A_{1} t+A_{2} t^{2}+i_{1} \overline{R(t)}
$$

où les $A$ et $\lambda$ sont des fonctions de $z$.

On déduit alors de l'énoncé de M. Fucis, cité plus laaut, la condition suivante

$$
\frac{d R}{d z}+\frac{d R}{d t}\left(A_{0}+A_{1} t+A_{2} t^{2}\right)=\left(B_{0}+B_{1} t\right) R(t)
$$

$B_{0}$ et $B_{1}$ étant des fonctions de $z$.

On peut pousser plus loin encore l'étude de cette condition à laquelle s'arrète le célèbre analyste que non citons.

Soit:

$$
R(t)=(t-\alpha)(t-\beta)(t-\gamma)(t-o)
$$

$\alpha, \beta, \gamma$ et $\partial$ étant des fonctions de $z$. Posons:

$$
t=\frac{a u+b}{c u+d}
$$

$a, b, c, d$ étant des fopctions de $z$ que nous déterminerons plus complètement dans la suite et auxquelles nous imposerons d'abord la condition:

$$
a d-b c=1 \text {. }
$$

Les équations (1), (2) et (3) vont se transformer. En posant:

$$
\alpha=\frac{a a^{\prime}+b}{c a^{\prime}+d}, \quad \beta=\frac{a \beta^{\prime}+b}{c \beta^{\prime}+d}, \quad \gamma=\frac{a \gamma^{\prime}+b}{c \gamma^{\prime}+d}, \quad \delta=\frac{a r^{\prime}+b}{c \sigma^{\prime}+d}
$$

on aura:

$$
R(t)=\frac{\left(u-u^{\prime}\right)(u-\beta)\left(u-\gamma^{\prime}\right)\left(u-o^{\prime}\right)}{(c u+d)^{4}\left(c a^{\prime}+d\right)(c \beta+d)\left(c \gamma^{\prime}+d\right)\left(c \sigma^{\prime}+\bar{d}\right)}=\frac{R_{1}}{(c u+d)^{i} \bar{M}}
$$

$M$ étant une fonction de $z$,

$$
A_{0}+A_{1} t+A_{2} t^{2}=\frac{A_{0}^{\prime \prime}+A_{1}^{\prime \prime} u+A_{2}^{\prime \prime} u^{2}}{(c u+d)^{2}}, \quad B_{0}+B_{1} t=\frac{B_{0}^{\prime}+B_{1}^{\prime} u}{c u+d}
$$


les $A^{\prime \prime}$ et les $B^{\prime}$ étant des fonctions de $z$. Il vient ensuite

d'où, en posant,

$$
\frac{d t}{d z}=\frac{d u}{d z(c u+d)^{2}}+\frac{c_{0}+c_{1}^{1} u+C_{2} u^{2}}{(c u+d)^{2}}
$$

$$
A_{0}^{\prime \prime}-C_{0}^{\prime}=A_{0}^{\prime}, \quad A_{1}^{\prime \prime}-C_{1}^{\prime}=A_{1}^{\prime}, \quad A_{2}^{\prime \prime}-C_{2}^{\prime}=A_{2}^{\prime}
$$

on tirera:

$$
\frac{d u}{d z}=A_{0}^{\prime}+A_{1}^{\prime} u+A_{2}^{\prime} u^{2}+\lambda_{1} \overline{R_{1}}
$$

et on trouverait aisément:

$$
\frac{d R_{1}}{d z}+\frac{d R_{1}}{d u}\left(A_{0}^{\prime}+A_{1}^{\prime} u+A_{2}^{\prime} \iota^{2}\right)=\left(B_{0}^{\prime}+B_{1}^{\prime} u\right) R_{1} .
$$

Ainsi la forme des équations (1), (2) et (3) n'est pas changée par la transformation (4), ce qu'il était d'ailleurs facile de prévoir.

Nous déterminerons $a, b, c, d$ en fonction de $z$ par les conditions:

$$
d \alpha-b=(c+d) \beta-(a+b)=(d-c) \gamma-(b-a)=0
$$

qu'il est toujours possible de remplịr et qui entraìnent:

$$
\alpha^{\prime}=0, \quad \beta^{\prime} \doteq \mathrm{I}, \quad \gamma^{\prime}=-\mathrm{I} .
$$

D'où la conclusion suivante:

Il est toujours permis de supposer:

$$
R(t)=t\left(t^{2}-1\right)(t-\partial)
$$

$o$ étant une fonction de $z$ qui.définit le module des fonctions elliptiques engendrées par $\sqrt{R}$. C'est l'hypothèse que nous ferons désormais.

L'équation (2) devient alors:

$$
t\left(1-t^{2}\right) \frac{d \grave{\partial}}{d z}+\frac{d R}{d t}\left(A_{0}+A_{1} t+A_{2} t^{2}\right)=\left(B_{0}+B_{1} t\right) R
$$

Faisons successivement dans cette équation:

$$
t=\mathrm{o}, \quad t=\mathrm{I}, \quad t=-\mathrm{I},
$$


elle deviendra:

$$
\frac{d R}{d t}\left(A_{0}+A_{1} t+A_{2} t^{2}\right)=0, \quad(t=- \text { I }, \text { о. I }) .
$$

Mais $\frac{d R}{d t}$ ne saurait s'annuler pour une de ces trois valeurs de $t$, sans quoi $\partial$ serait égal à $-\mathrm{I}$, ̀̀ $\mathrm{o}$, ou ̀̀ $\mathrm{I}$, et le nombre $p$ ne serait plus égal à $\mathrm{I}$, mais à $\mathrm{O}$. On a donc:

$$
A_{0}+A_{1} t+A_{2} t^{2}=\mathrm{O}, \quad(t=-\mathrm{I}, \mathrm{O}, \mathrm{I})
$$

d'où

$$
A_{0}=A_{1}=A_{2}=\mathrm{o} \text {. }
$$

L'équation (2) se réduit alors à:

$$
t\left(\mathrm{I}-t^{2}\right) \frac{d i}{d z}=\left(B_{0}+B_{1} t\right) R
$$

Si dans cette équation on fait $t=i$, il reste:

$$
\frac{d \grave{d}}{d z}=0 \text {. }
$$

Ainsi $\partial$ est une constante.

$\mathrm{Si}$ on regarde un instarit $z$ comme une constante, l'équation (A) devient une relation algébrique de genre $p$. Cette relation définit une certaine surface de Riemaxis $S$ qui dépend de $z$. Ici $p=1$; donc à chacune de ces surfaces de Ricmaxi correspond un système de fonctions elliptiques et le module de ces fonctions pourra s'appeler le module de la surface $S$.

$I l$ résulte de ce qui précède que le module de la surface $S$ est invariable.

Cela posé, l'équation (I) devient:

$$
\frac{d t}{d z}=\lambda_{1} K
$$

ou:

$$
\frac{d t}{\sqrt{R}}=\lambda d z
$$

$R$ ne dépend que de $t$ et $\lambda$ ne dépend que de $z$; les variables sont donc séparées. 
Posons $\lambda=\frac{d \mu}{d z}, \mu$ étant une fonction de $z$. I.inversion de la relation

donnera:

$$
\frac{d t}{\sqrt{R}}=d / l
$$

$$
t=c(\mu+c)
$$

$\varphi$ étant l'algorithme d'une fonction doublement périodique et $c$ étant la constante d'intégration.

Les points singuliers de la fonction $t$, seront ceux de la fonction $\mu$; ils seront done indépendants de la constante d'intégration et seront les mèmes pour toutes les intégrales.

Ainsi dans le cas de $p=\mathrm{I}$, comme dans celui de $p=0$, nous ne sommes pas conduits à une classe réellement nourelle d'équations différentielles.

Il reste à examiner le cas de $p>\mathrm{I}$, laissé de cóté par M. Fuchs. Une petite digression sur les surfaces de Rrmasx est ici nécessaire. Soit:

$$
f_{0}\left(y_{0}, y_{0}^{\prime}\right)=0
$$

une relation algébrique de genre $p$, définissant une surface de Ricuars $S_{0}$. Soit

$$
f_{1}^{\prime}\left(y_{1}, y_{1}^{\prime}\right)=0
$$

une relation de mème genre définissant une surface de Rimaxx $S_{1}$.

Les deux surfaces $S_{0}$ et $S_{1}$ seront dites équivalentes si l'on peut passer de l'une à l'autre par une transformation birationnelle, c'est à dire en établissant entre les deux points analytiques $\left(y_{0}, y_{0}^{\prime}\right),\left(y_{1}, y_{1}^{\prime}\right)$, une relation telle que $y_{1}$ et $y_{1}^{\prime}$ puissent s'exprimer rationnellement en fonctions de $y_{0}$ et $y_{0}^{\prime}$; et réciproquement.

On sait qu’il y a certains invariants qui ne sont pas altérés par les tranşformations birationnelles; ce sont les modules. Il y a $3 p-3$ modules pour une surface de Ricuaxs de genre $p>1$ et I module pour une surface de genre 1 . Deux surfaces de Ricmaxs équivalentes ont donc mêmes modules.

Reprenons maintenant l'équation (A) et considérons-la comme représentant une surface de Ricmaxi $S$ variable avec $z$. Je dis que les modules de cette surface $S$ seront constants et indépendants de $z$. 
En effet partons de la valeur initiale $z_{0}$ de $z$, à laquelle correspond une certaine surface de Riemans $S_{0}$. Soient $y_{0}$ et $y_{0}^{\prime}$ les valeurs initiales d'une certaine intégrale de l'équation (A) et de sa dérivée; le point analytique $\left(y_{0}, y_{0}^{\prime}\right)$ appartiendra à la surface $S_{0}$.

Allons ensuite du point $z_{0}$ au point $z_{1}$ en suivant un chemin déterminé. La surface de Riemann que nous avons appelée $S$ et qui pour $z=z_{0}$. se réduisait à $S_{0}$, variera avec $z$ et pour $z=z_{1}$, se réduira à $S_{1}$. Pour $z=z_{1}$, l'intégrale considérée et sa dérivée se réduiront à $y_{1}$ et $y_{1}^{\prime}$ et le point analytique $\left(y_{1}, y_{1}^{\prime}\right)$ appartiendra à la surface $S_{1}$.

Faisons maintenant varier sur la surface $S_{0}$ le point analytique $\left(y_{0}, y_{0}^{\prime}\right)$ qui définit les valeurs initiales correspondant à l'intégrale envisagée, mais conservons des valeurs invariables à $z_{0}$ et $\dot{a} z_{1}$ et ne faisons pas varier non plus le chemin qui mène de $z_{0}$ à $z_{1}$. Dans ces conditions, les surfaces $S_{0}$ et $S_{1}$ ne varieront pas, mais l'intégrale considérée variera et dépendra des valeurs initiales $y_{0}$ et $y_{0}^{\prime}$ que l'on aura choisies. Par conséquent $y_{1}$ et $y_{1}^{\prime}$ seront des fonctions de $y_{0}$ et de $y_{0}^{\prime}$.

$\mathrm{Je}$ dis que ce seront des fonctions uniformes et continues du point analytique $\left(y_{0}, y_{0}^{\prime}\right)$. En effet si l'on se donne les valeurs initiales $y_{0}$ et $y_{0}^{\prime}$, l'intégrale qui correspondra à ces valeurs initiales sera entièrement déterminée. Cette intégrale considérée comme fonction de $z$, peut prendre pour $z=z_{1}$ des valeurs différentes. Mais parmi elles, il y en a une, qui est celle que nous avons appelée $y_{1}$ et qui est celle que l'on obtient en allant du point $z_{0}$ au point $z_{1}$ par le chemin particulier que nous avons choisi. Cette valeur $y_{1}$ ainsi définie est parfaitement déterminée. C'est donc une fonction uniforme du point analytique $\left(y_{0}, y_{0}^{\prime}\right)$.

Cette fonction uniforme pourrait toutefois être discontinue. Voyons comment cela pourrait arriver, par un exemple simple. Reprenons l'équation:

$$
z d z+y d y=0
$$

et son intégrale:

$$
y=\sqrt{c^{2}-z^{2}}
$$

Soit $z_{0}=0, z_{1}=\mathrm{I}$ et allons du point $\mathrm{O}$ au point $\mathrm{I}$ par la droite qui joint ces deux points. Il viendra:

$$
y_{0}=c
$$

Acta mathematica. 7. Imprimé le 18 Février 1885. 
et

$$
y_{1}= \pm \sqrt{c^{2}-\mathrm{I}}= \pm \sqrt{y_{0}^{2}-\mathrm{I}} .
$$

Ainsi $y_{1}$ est exprimé en fonction de $y_{0}$. Il reste toutefois pour le définir complètement à décider si l'on doit prendre le signe + ou le signe Supposons d'abord que la partie imaginaire de $y_{0}$ soit positive. Posons:

$$
2 y_{0}=\left(t+\frac{1}{t}\right) \cos \varphi+i\left(t-\frac{1}{t}\right) \sin \varphi
$$

$t$ et $\varphi$ étant des quantités réelles et telles que

$$
0 \leqq \varphi<2 \pi, \quad t \geqq 1 .
$$

Cela est toujours possible et d'une seule manière, sauf une exception dont nous parlerons plus loin. Il viendra:

$$
2 \sqrt{y_{0}^{2}-\mathrm{I}}= \pm\left[\left(t-\frac{\mathrm{I}}{t}\right) \cos \varphi+i\left(t+\frac{\mathrm{I}}{t}\right) \sin \varphi\right] \text {. }
$$

Cela posé, pour déterminer le signe qu'il faut prendre, faisons varier $z$ de $O$ a $I$, en suivant la droite qui joint ces deux points.

Nous écrivons:

$$
\begin{gathered}
2 y_{0}=\left(u+\frac{z^{3}}{u}\right) \cos \psi+i\left(u-\frac{z^{2}}{u}\right) \sin \psi \\
0 \leqq \psi<2 \pi, \quad u \geqq z ;
\end{gathered}
$$

c' devra se réduire à $\varphi$ et $u$ à I pour $z=\mathrm{I}$. Il viendra:

$$
2 \sqrt{y_{0}^{2}}-\overline{z^{2}}= \pm\left[\left(u-\frac{z^{2}}{u}\right) \cos \phi+i\left(u+\frac{z^{2}}{u}\right) \sin \phi\right]
$$

et comme cette expression devra se réduire à $2 y_{0}$ pour $z=0$, il faudra prendre le signe + et il viendra:

$$
2 y_{1}=\left(t-\frac{\mathrm{I}}{t}\right) \cos \varphi+i\left(t+\frac{\mathrm{I}}{t}\right) \sin \varphi \text {. }
$$

Cette expression n'est pas une fonction continue de $y_{0}$. Soit en effet

$$
t=\mathrm{I}+\Sigma
$$


$\varepsilon$ étant infiniment petit. Les deux valeurs de $2 y_{0}$ :

$$
\left(\mathrm{I}+\varepsilon+\frac{\mathrm{I}}{\mathrm{I}+\varepsilon}\right) \cos \varphi+i\left(\mathrm{I}+\varepsilon-\frac{\mathrm{I}}{\mathrm{I}+\varepsilon}\right) \sin \varphi=2 \cos \varphi+\text { inf. petit. }
$$

et

$\left(\mathrm{I}+\varepsilon+\frac{\mathrm{I}}{\mathrm{I}+\varepsilon}\right) \cos (-\varphi)+i\left(\mathrm{I}+\varepsilon-\frac{\mathrm{I}}{\mathrm{I}+\varepsilon}\right) \sin (-\varphi)=2 \cos \varphi+$ inf. petit.

sont infiniment voisines, tandis que les valeurs correspondantes de $2 y_{1}$ :

$$
\left(\mathrm{I}+\varepsilon-\frac{\mathrm{I}}{\mathrm{I}+\varepsilon}\right) \cos \varphi+i\left(1+\varepsilon+\frac{\mathrm{I}}{\mathrm{I}+\varepsilon}\right) \sin \varphi=2 i \sin \varphi+\text { inf. petit. }
$$

ct

$\left(\mathrm{I}+\varepsilon-\frac{\mathrm{I}}{\mathrm{I}+\varepsilon}\right) \cos (-\varphi)+i\left(\mathrm{I}+\varepsilon+\frac{\mathrm{I}}{\mathrm{I}+\varepsilon}\right) \sin (-\varphi)=-2 i \sin \varphi+$ inf. petit ne sont pas infiniment voisines comme elles devraient l'ètre si $y_{1}$ était fonction continue de $y_{0}$.

A quoi tient.ce fait? Supposons que $y_{0}$ soit réel et compris entre - I et $+\mathrm{I}$. Alors il faudra prendre $t=\mathrm{I}$. Et pour l'angle $\varphi$ nous aurons deux valeurs distinctes satisfaisant toutes deux à la condition:

$$
\cos \varphi=y_{0} \text {. }
$$

D'ailleurs rien dans les hypothèses faites jusqu'ici, ne nous permettra de décider entre ces deux valeurs de $\varphi$ qui conduisent pour $y_{1}$ à deux valeurs égales et de signe contraire. Rendons-nous compte de la raison d'être de cette anomalie. Supposons que nous ayons donné à $y_{0}$ une valeur réelle comprise entre - I et $+_{I}$. L'intégrale correspondante

$$
y=\sqrt{y_{0}^{2}-z^{2}}
$$

présentera un point de ramification

$$
z=\left|y_{0}\right|
$$

situé sur la droite qui joint le point $z=0$ au point $z=\mathrm{I}$, c'est à dire sur le chemin même que nous sommes convenus de suivre pour aller du premier de ces points au second. Quand la variable $z$, en suivant cé 
chemin, aura franchi ce point de ramification, rien dans les hypothèses faites ne nous permettra de décider quel signe il faut attribuer au radical $\sqrt{y_{0}^{2}-z^{2}}$.

Je suis entré dans d'assez longs détaits sur ce cas simple et j'espère avoir fait comprendre comment $y_{1}$ pourrait être une fonction discontinue de $y_{0}$.

Cela arriverait si l'un des points du chemin que nous suivons pour aller de $z_{0} \quad \dot{a} z_{1}$ était un point de ramification pour l'une des intégrales. Mais rien de pareil n'est à craindre dans le cas qui nous occupe. Nous avons supposé en effet que les points de ramification étaient les mêmes pour toutes les intégrales et par conséquent qu'il ne pouvait y avoir de points de ramification des intégrales que pour certaines valeurs particulières de $z$.

Or nous aurons toujours pu choisir le chemin qui va de $z_{0}$ ì $z_{1}$ de telle sorte qu'il ne passe par aucune de ces valeurs particulières.

Donc $y_{1}$ est une fonction uniforme et continue de $y_{0}$ et $y_{0}^{\prime}$.

Il est aisé de voir que cette fonction n'a d'autres singularités que des pôles.

Donc $y_{1}$ est une fonction rationnelle de $y_{0}$ et $y_{0}^{\prime}$.

Pour la mêrne raison, $y_{1}^{\prime}$ est une fonction rationnelle de $y_{0}$ et $y_{0}^{\prime}$; et de même $y_{0}$ et $y_{0}^{\prime}$ sont des fonctions rationnelles de $y_{1}$ et $y_{1}^{\prime}$.

Donc on peut passer de $S_{0}$ à $S_{1}$ par une transformation birationnelle.

Donc ces deux surfaces de Ribmaxy ont mèmes modules.

Donc les modules de la surface $S$ sont indépendants de $z$.

C. Q. F. D.

C'est le résultat que nous avions obtenu plus hant pour le cas de $p=\mathrm{I}$ et qui est étendu ainsi au cas général.

Pour pousser plus loin cette étude, il est nécessaire de dire quelques mots des transformations birationnelles des surfaces de RifmanN en ellesmêmes.

Une surface de genre o peut se transformer en elle-mème par une infunité de transformations birationnelles formant un groupe continu à trois paramètres. Soit en effet

$$
f(x, y)=\mathrm{o}
$$


une relation algébrique de genre o et $\boldsymbol{S}$ la surface de Riemann correspondante, on posera:

$$
x=\varphi(t), \quad y=\phi(t)
$$

$\varphi$ et $\psi$ étant rationnels. Si ensuite on pose

$$
t^{\prime}=\frac{\alpha t+\beta}{\gamma t+\delta}
$$

$\alpha, \beta, \gamma$ et $\partial$ étant des constantes quelconques, puis:

$$
x^{\prime}=\varphi\left(t^{\prime}\right), \quad y^{\prime}=\psi\left(t^{\prime}\right)
$$

$x^{\prime \prime}$ et $y^{\prime}$ seront fonctions rationnelles de $x$ et $y$ et réciproquement, on aura ainsi une triple infinité de transformations birationnelles de la surface $S$ en elle-mème.

Les transformations birationnelles d'une surface de genre I en ellemème forment encore un groupe continu, mais ce groupe ne contient plus qu'un seul paramètre. Supposons en effet que la relation (5) et par conséquent la surface $S$ soit de genre $\mathrm{r}$ et non plus de genre $o$. Nous poserons:

$$
x=\varphi(t), \quad y=\varphi(t)
$$

$\varphi$ et $\psi$ étant des fonctions doublement périodiques avec les périodes $\omega$ et $\omega^{\prime}$. Soient

$$
x^{\prime}=\varphi\left(t^{\prime}\right), \quad y^{\prime}=\psi\left(t^{\prime}\right)
$$

un autre système de valeurs satisfaisant à la relation (5) et supposons que $x^{\prime}$ et $y^{\prime}$ puissent s'exprimer rationnellement en $x$ et $y$, et réciproquement. On verra:

$1^{\circ}$ que $t^{\prime}$ est une fonction entière de $t$.

$2^{\circ}$ que $t$ est une fonction entière de $t^{\prime}$.

$3^{\circ}$ que l'on a entre $t$ et $t^{\prime}$ une relation de la forme:

$$
\alpha t+\beta t^{\prime}+\gamma=0
$$

$\alpha, \beta$ et $\gamma$ étant des constantes.

$4^{\circ}$ que lorsque $t$ augmente d'une période, $t^{\prime}$ doit augmenter aussi d'une période et réciproquement. Si par exemple $t$ augmente de $\omega, t^{\prime}$ 
devra augmenter de $m \omega+n \omega^{\prime}$, $m$ et $n$ étant des entiers. Il vient donc:

et de mème:

$$
\alpha \omega+\beta\left(n \omega+n \omega^{\prime}\right)=0
$$

$$
\begin{aligned}
& \alpha \omega^{\prime}+\beta\left(m^{\prime} \omega+n^{\prime} \omega^{\prime}\right)=0 \\
& \alpha\left(m_{1} \omega+n_{1} \omega^{\prime}\right)+\beta \omega=0 \\
& \alpha\left(m_{1}^{\prime} \omega+n_{1}^{\prime} \omega^{\prime}\right)+\beta \omega=0 .
\end{aligned}
$$

Les deux dernières équations sont des conséquences des deux premières pourvu que l'on suppose: $m n^{\prime}-m^{\prime} n=\mathbf{I}$. Cette condition est d'ailleurs nécessaire pour que les quatre équations soient compatibles. Nous ronplacerons donc nos quatre équations par les trois suivantes:

$$
\begin{gathered}
(\alpha+\beta m) \omega+\beta n \omega^{\prime}=\beta m^{\prime} \omega+\left(\alpha+\beta n^{\prime}\right) \omega^{\prime}=0 \\
m n^{\prime}-m m^{\prime} n=1 .
\end{gathered}
$$

Ces équations peuvent ètre satisfaites de deux manières:

$I^{\circ}$. En faisant

$$
\alpha=\mathrm{I}, \quad \beta=-\mathrm{I}, \quad m=n^{\prime}=\mathrm{I} ; \quad m^{\prime}=n=0
$$

d'où:

$$
t^{\prime}=t+r
$$

On est ainsi conduit à une simple infinité de transformations de la surface $S$ en elle-mème, dépendant d'un seul paramètre $\gamma$ et formant un groupe continu.

$2^{\circ}$. Le rapport $\frac{\alpha}{\beta}$ est donné par l'équation:

$$
\alpha^{2}+\beta^{2}+\alpha \beta\left(m+n^{\prime}\right)=0 .
$$

De plus ce rapport ne doit pas ètre réel, (si on laisse de cóté le cas que nóus venons de traiter et celui que nous allons traiter plus loin) sans quoi le rapport $\frac{\omega}{\omega^{\prime}}$ serait lui-même réel. On devra donc avoir:

$$
m+n^{\prime}=0, \text { I ou }- \text { I. }
$$


Il est aisé de déduire de là que le rapport $\frac{\omega}{\omega^{\prime}}$ doit avoir une des valeurs

$$
\frac{\lambda e^{\frac{k \pi \pi}{6}}+\mu}{\lambda e^{\frac{k i \pi}{3}}+\mu^{\prime}}
$$

$k, \lambda, \mu, \lambda^{\prime}, \mu^{\prime}$ étant des entiers tel que

$$
\lambda \mu^{\prime}-\lambda^{\prime} \mu=\mathrm{I}, \quad k \equiv(2,3,4,8,9 \text { ou } \mathrm{I0})(\bmod \text { I } 2) .
$$

Ce cas ne pourra donc se présenter que pour certaines valeurs particulières du module de la surface $S$. Pour ces valeurs, la surface $S$ admettra outre le groupe continu trouvé plus haut, une autre transformation dont la puissance $3^{\mathrm{e}}, 4^{\mathrm{e}}$ ou $6^{\mathrm{e}}$ se confondra avec la substitution identique, ainsi que les diverses puissances de cette transformation et les combinaisons de ces puissances avec les diverses transformations du groupe continu.

$3^{0}$. On peut enfin satisfaire à nos trois équations en faisant:

$$
\begin{aligned}
t & =-t^{\prime} \\
\alpha=\mathrm{I}, \quad \beta=\mathrm{I}, \quad m & =n^{\prime}=-\mathrm{I}, \quad m^{\prime}=n=0 .
\end{aligned}
$$

On est ainsi conduit $\dot{a}$ une transformation $T$ de la surface $S$ en ellemème. Si l'on appelle $\tau$ une transformation quelconque du groupe continn trouvé plus haut, toutes les transformations birationnelles de la surface $S$ en elle-même sont comprises dans l'une des formules

$$
\tau \text { et } \tau T \text {. }
$$

Il n'y a d'exception que si le rapport $\frac{\omega}{\omega^{\prime}}$ prend l'une des valeurs (6). Les considerations qui précédent ne présentent aucune difficulté, je les ai pourtant développées avec détail parce que jai l'intention d'appliquer une méthode tout à fait analogue à la recherche des transformations des surfaces de genre $p>\mathrm{I}$.

Ces surfaces ne peuvent être: transformées en elles-mêmes que d'un nombre fini de manières.

Ce théorème était soupçonné depuis longtemps, mais la démonstration 
II. Poincarí.

a longtemps arrêté les géomètres. Elle a été trouvée il y a quelques années par M. KLEIN.

Voici en effet ce que ce géomètre me fit l'honncur de m'écrire à la date du 3 Avril I 882.

Eine Reihe von Theoremen über algebraische Functionen beweisı man vermöge der neuen $\eta$ Function sofort (fonction intimement liée aux fonctions fuchsiennes). Zum Beispiel den Satz, den ich in meiner Schrift über Rremani nur erst als wahrscheinlich bezeichnete, dass nämlich eine Fläche $p>$ I niemals unendlich viele discrete eindeutige Transformationen in sich besitzen kann (vermöge deren sie in eine $\infty$ Zahl äquivalenter. Fundamentalpolygone zerlegt erscheinen würde).

Il est facile de reconstituer dans tous ses détails la démonstration de M. Kuein.

Reprenons en effet la relation (5) et supposons que cette relation et par conséquent la surface $S$ soient de genre $p>$ I. Nous poserons:

$$
x=\varphi(t), \quad y=\psi(t)
$$

$\varphi(t)$ et $\phi(t)$ étant des fonctions fuchsiennes dont le polygone générateur $R_{0}$ aura $4 p$ côtés, les côtés opposés étant conjugués. Tous les sommets formeront un seul cycle et la somme des angles sera égale à $2 \pi$ (cf. Théorie des groupes fuchsiens, Acta Mathematica, T. I, p. 23 et 42; et Mémoire sur les fonctions fuchsiennes, Acta Mathematica, T. I, p. 256 et suiv.). Cela est toujours possible (cf. Mémoive sur les groupes des équations linéaires, Acta Mathematica, T. 4, p. 272 et 276).

Soient:

$$
x^{\prime}=\varphi\left(t^{\prime}\right), \quad y^{\prime}=\phi\left(t^{\prime}\right)
$$

et supposons que $x^{\prime}$ et $y^{\prime}$ soient fonctions rationnelles de $x$ et de $y$, et réciproquement. Qu'arrivera-t.il si l'on étudie $t^{\prime}$ comme fonction de $t$ ? En premier lieu, tant que $t$ reste intérieur au cercle fondamental, $t^{\prime}$. est une fonction holomorphe de $t$.

De plus $t^{\prime}$ reste intérieur au cercle fondamental.

Réciproquement quand $t^{\prime}$ reste intérieur au cercle fondamental, $t$ est fonction holomorphe de $t^{\prime}$ et reste intérieur au cercle fondamental. 
On en déduit aisément (cf. Mémoire sur les groupes des équations linéaires, p. 23I) que

$$
t^{\prime}=\frac{\alpha t+\beta}{\gamma t+\delta}
$$

la substitution:

$$
\sigma=\left(t, \frac{a t+\beta}{i t+i}\right)
$$

conservant le cercle fondamental.

Soit maintenant $G$ le groupe des fonctions fuchsiennes $\varphi(t)$ et $\phi^{\prime}(t)$. Je dis qu'il est permutable à la substitution $\sigma$. Soit en effet $\tau$ une substitution quelconque du groupe $G$ : je dis que $\sigma^{-1} \tau \sigma$ fera aussi partic de ce groupe. En effet, $\tau$ faisant partie de $G$, on aura:

$$
x=\varphi(t)=\varphi(t . \tau), \quad y=\phi(t)=\phi(t . \tau)
$$

d'où :

$$
R[\varphi(t), \phi(t)]=R[\varphi(t . \tau), \phi(t . \tau)]
$$

$R$ étant l'algorithme d'une fonction rationnelle quelconque. D'autre part on a, par hypothèse:

et de mème

$$
\begin{aligned}
& x^{\prime}=\varphi(t . \sigma)=R_{1}[\varphi(t), \phi(t)] \\
& y^{\prime}=\psi(t . \sigma)=R_{2}\left[\varphi(t), \varphi^{\prime}(t)\right]
\end{aligned}
$$

$$
\begin{aligned}
& \varphi(t . \tau . \sigma)=R_{1}[\varphi(t . \tau), \phi(t . \tau)] \\
& \psi(t . \tau . \sigma)=R_{2}[\varphi(t . \tau), \psi(t . \tau)]
\end{aligned}
$$

d'où:

$$
\varphi(t \tau \sigma)=\varphi(t \sigma), \quad \phi(t \tau \sigma)=\psi(t \sigma)
$$

ou en changeant $t$ en $t \sigma^{-1}$

$$
\varphi\left(t \sigma^{-1} \tau \sigma\right)=\varphi(t), \quad \psi\left(t \sigma^{-1} \tau \sigma\right)=\phi(t) .
$$

Donc la substitution $\sigma^{-1} \tau \sigma$ fait partie du groupe $G$.

C. Q. F. D.

Les substitutions linéaires permutables au groupe $G$ et conservant le cercle fondamental forment un groupe $G^{\prime}$. Ce groupe $G^{\prime \prime}$ contient Acta mathematica. 7. Imprimé le 24 Fétrier 1885. 
évidemment le groupe $G$; en d'autres termes $G$ est un sous-groupe de $G^{\prime}$, et on voit aisément qu'à toute substitution de $G^{\prime}$, n'appartenant pas à $G$, correspondra une transformation birationnelle de la surface $S$ en elle-mème.

Remarquons de plus que d'après la définition même de $G^{\prime}, G$ est un »sous-groupe distingué» de $G^{\prime}$.

Mais il y a deux espèces de sous-groupes: les sous-groupes d'indice fini (qui sont tels qu'on obtient toutes les substitutions du groupe principal, en prenant la résultante des diverses substitutions du sous-groupe et d'un nombre fini d'autres substitutions) et les sous-groupes d'indice infini.

Je me propose de démontrer que $G$ est un sous-groupe d'indice fini, et par conséquent que la surface $S$ n'admet qu'un nombre fini de transformations birationnelles en elle-mème.

J'établirai d'abord que $G^{\prime}$ est un groupe fuchsien, c'est à dire un groupe proprement discontinu. En effet s'il ne l'était pas, il serait ou bien continu (c'est à dire qu'il contiendrait des substitutions infinitésimales), ou bien improprement discontinu. (Cf. Théorie des groupes kleinéens, Acta Mathematica, T. 3, p. 57.)

$I^{\circ}$. Il ne peut pas être continu; car s'il contenait une substitution infinitésimale $\sigma$, cette substitution serait permutable, non seulement au groupe $G$, mais i toutes les substitutions de ce groupe.

Soient en effet

$$
\tau_{1}, \tau_{2}, \ldots, \tau_{2 p}
$$

les substitutions fondamentales de $G$. D'après les hypothèses faites, les substitutions

$$
\sigma^{-1} \tau_{1} \sigma, \sigma^{-1} \tau_{2} \sigma, \ldots, \sigma^{-1} \tau_{2 p} \sigma
$$

feront également partie du groupe $G$. Mais ces substitutions diffèrent infiniment peu de

$$
\tau_{1}, \tau_{2}, \ldots, \tau_{2 p}
$$

puisque $\sigma$ est infinitésimale. Mais le groupe $G$ étant discontinu ne devra contenir aucune substitution infinitésimale, il ne pourra donc contenir deux substitutions distinctes, mais différant infiniment peu l'une de l'autre. Donc on a:

$$
\tau_{i}=\sigma^{-1} \tau_{i} \sigma, \quad(i=1,2, \ldots, 2 p) .
$$

Donc $\sigma$ est permutable aux substitutions fondamentales de. $G$; elle est 
donc permutable à toutes les substitutions de ce groupe, qui n'en sont que des combinaisons.

Mais je dis qu'on ne saurait trouver de substitution linéaire permutable à toutes les substitutions de $G$. En effet pour que deux substitutions linéaires $\Sigma^{\prime}$ et $\Sigma^{\prime}$ soient permutables, il faut et il suffit, ou bien qu'elles aient mêmes points doubles (les deux points doubles pouvant dans certains cas se confondre en un seul), ou bien qu'elles aient toutes deux pour multiplicateur - I et que les quatre points doubles soient conjugués harmoniques.

Nous n'avons pas besoin de nous inquiéter de ce second cas de permutabilité. En effet une substitution infinitésimale ne peut avoir pour multiplicateur - I. Si donc le groupe $G^{\prime}$ contenait une substitution infinitésimale $\sigma$, toutes les substitutions de $G$ devraient avoir mêmes points doubles que $\sigma$; elles seraient donc toutes permutables entre elles, ce qui n'a pas lieu.

Donc $G^{\prime}$ ne peut être continu.

$2^{\circ}$. $G^{\prime}$ ne peut pas non plus ètre improprement discontinu. J'ai démontré en effet (Groupes kleinéens, p. 58) que tout groupe formé de substitutions linéaires conservant le cercle fondamental, et ne contenant pas de substitution infinitésimale, est proprement discontinu à l'intérieur $\mathrm{du}$ cercle fondamental.

Donc $G^{\prime}$ est un groupe fuchsien.

Il aura donc un polygone générateur $R_{0}^{\prime}$, et l'indice de $G$ considéré comme sous-groupe de $G^{\prime}$ sera égal à la $S$ de $R_{0}$ (polygone générateur de $G$ ) divisée par la $S$ de $R_{0}^{\prime}$. (Cf. Mémoire sur les groupes des équations linéaires, Acta Mathematica, T. 4, p. 285.) Or la $S$ de $R_{0}$ est finie, donc $G$ est un sous-groupe d'indice fini.

C. Q. F. D.

En général, le groupe $G^{\prime}$ ne diffère pas du groupe $G$, de sorte que la surface $S$ n'admet aucune transformation birationnelle en elle-méme. Elle ne peut en avoir que dans des cas exceptionnels qui correspondent évidemment aux différents cas de symétrie que peut présenter le polygone $R_{0}$.

Soient maintenant deux surfaces de Ricmand $S_{0}$ et $S_{1}$, équivalentes, ct de genre $p$.

Si l'on peut passer de l'une à l'autre par deux transformations 
birationnelles $T$ et $U$, la transformation $T U^{-1}$ changera en elle-mème la surface $S_{0}$.

D'où les conclusions suivantes:

I $^{\circ}$. Si $p=0$, on peut passer de $S_{0}$ i $S_{1}$ par une triple infinité de transformations birationnelles.

$2^{\circ}$. Si $p=\mathrm{I}$, on peut passer de $S_{0}$ à $S_{1}$ par une simple infinité de transformations birationnelles.

$3^{\circ}$. Si $p>1$, il n'y a en général qu'une seule transformation birationnelle qui permette de passer de $S_{0}$ ì $S_{1}$, et il n'y en a jamais qu'un nombre fini.

Gràce $\dot{a}$ ces propositions, il est aisé de retrouver les résultats que uous avons démontrés plus haut pour les cas de $p=0$ et de $p=\mathrm{I}$ et de traiter complètement le cas de $p>1$.

Reprenons en effet l'équation:

$$
F\left(y, y^{\prime}, z\right)=0
$$

et soit d'abord $p=0$. On pourra poser:

$$
y=\varphi(t), \quad y^{\prime}=\varphi(t)
$$

$\varphi$ et $\psi$ étant des fonctions rationnelles de $t$ dont les coëfficients dépendent de $z$.

Soit $y_{0}=\varphi\left(t_{0}\right), y_{0}^{\prime}=\phi\left(t_{0}\right)$ les valeurs initiales d'une certaine intégrale et de sa dérivée pour $z=z_{0}$, et

$$
y_{1}=\varphi\left(t_{1}\right), \quad y_{1}^{\prime}=\phi\left(t_{1}\right)
$$

les valeurs de cette $\cdot$ même intégrale et de sa dérivée pour $z=z_{1}$. On a vu que $y_{1}$ et ' $y_{1}^{\prime}$ doivent être fonctions rationnelles de $y_{0}$ et $y_{0}^{\prime}$ et réciproquement. On aura donc

$$
t_{1}=\frac{\alpha t_{0}+\beta}{\gamma t_{0}+\beta}
$$

Les coëfficients de cette substitution linéaire $\alpha, \beta, \gamma, \delta$ dépendent évidemment de $z_{0}$ et de $z_{1}$. Nous regarderons $z_{0}$ comıne une constante, et $z_{1}$ 
sera la variable indépendante; nous supprimerons donc l'indice $\mathrm{I}$ de $t_{1}$, $z_{1}, y_{1}, y_{1}^{\prime}$ et nous aurons

$$
t=\frac{a t_{0}+\beta}{\gamma t_{0}+\delta}
$$

où $t_{0}$ sera la constante d'intégration et ou $\alpha, \beta, i, \partial$ seront des fonctions de z. Si $\alpha^{\prime}, \beta^{\prime}, \gamma^{\prime}, o^{\gamma}$ sont les dérivées de ces fonctions; il viendra:

$$
\frac{d t}{d z}=\frac{\left(\alpha^{\prime} t_{0}+\beta^{\prime} \cdot\left(\gamma t_{0}+o\right)-\left(\gamma^{\prime} t_{0}+o^{\prime}\right)\left(\alpha t_{0}+\beta\right)\right.}{\left(\gamma t_{0}+\partial\right)^{2}} .
$$

En éliminant $t_{0}$ entre (6) et $(7)$ on retomberait sur l'équation de Riccs'rr, ce qui confirme le résultat obtenu plus haut.

$\mathrm{Si}$ on remplace $t$ par sa valeur (6) dans l'expression

$$
y=\varphi(t)
$$

on trouve pour l'intégrale générale de l'équation $(A)$ :

$$
y=R\left(t_{0}\right)
$$

$R$ étant une fonction rationnelle de la constante dintégration $t_{0}$ et dont les coëfficients dépendent de $z$.

Réciproquement si l'on a une fonction $y$ de $t_{0}$ et de $z$, rationnelle par rapport à $t_{0}$, et que l'on forme la dérivée $y^{\prime}=\frac{d y}{d z}$, on obtiendra par l'élimination de $t_{0}$ une équation différentielle de la forme (A) entre $y, y^{\prime}$ et $z$.

$\mathrm{Si}$ en particulier l'équation (A) est algébrique, non seulement par rapport à $y$ et à $y^{\prime}$, mais encore par rapport à $z$, l'équation de Ricca'TI à laquelle on sera conduit aura ses coëfficients algébriques en $z$, et par conséquent l'intégration de l'équation (A) sera ramenée à celle des équations linéaires du $2^{\mathrm{d}}$ ordre à coëfficients algébriques.

Supposons maintenant $p=\mathrm{I}$. Nous pourrons poser:

$$
y=\varphi(t), \quad y^{\prime}=\varphi^{\prime}(t)
$$

$\varphi$ et $\psi$ étant des fonctions doublement périodiques de $t$ dont les coëfticients dépendent de $z$. Conservons aux notations

$$
z_{0}, z_{1}, \quad y_{0}=\varphi\left(t_{0}\right), \quad y_{0}^{\prime}=\phi\left(t_{0}\right), \quad y_{1}=\varphi\left(t_{1}\right), \quad y_{1}^{\prime}=\psi\left(t_{1}\right)
$$


le mème sens que plus liaut. On sait que $y_{1}$ et $y_{1}^{\prime}$ doivent ètre des fonctions rationnelles de $y_{0}$ et $y_{0}^{\prime}$ et réciproquement. Donc d'après ce qu'on a vu plus haut sur les transformations des surfaces de genre i en elles mêmes, on devra avoir:

$$
t_{1}=\alpha t_{0}+\beta
$$

a étant. égal à l'une des quantités:

$$
\mathrm{I}, \quad-\mathrm{I}, \quad e^{\frac{2 k i \pi}{3}}, \quad-e^{\frac{2 k i \pi}{3}}, \pm i
$$

Comme $\alpha$ et $\beta$ doivent ètre des fonctions continues de $z_{1}$ et que $t_{1}$ doit se réduire à $t_{0}$ pour $z_{0}=z_{1}$, on aura

$$
\alpha=1, \quad t_{1}=t_{0}+\beta .
$$

Considérons $z_{0}$ comme constant, $z_{1}$ comme variable et supprimons l'indice I de $z_{1}, t_{1}, y_{1}, y_{1}^{\prime}$.

$\beta$ sera une fonction de $z$ et l'intégrale générale de l'équation (A) sera:

$$
y=\varphi\left(t_{0}+\beta\right)
$$

$\varphi$ étant une fonction doublement périodique dont les coêfficients dépendront de $z, \beta$ une fonction de $z$, et $t_{0}$ la constante d'intégration.

Si l'équation (A) est algébrique en $z$, les coëfficients de la fonction doublement périodique $\varphi(t)$ dépendent algébriquement de $z$; et la fonction $\beta$ de $z$ s'obtient par une simple quadrature. Posons:

$$
u=\operatorname{sn}(t)
$$

y sera une fonction algébrique de $u$ et de $z$, si l'équation (A) est algébrique en $z$. Si l'équation (A) n'est pas algébrique en $z$ et si elle s'écrit:

$$
\sum A_{m_{l}} y^{n} y^{\prime p}=0
$$

où les coëfficients $A_{m p}$ sont des fonctions non algébriques de $z, y$ sera une fonction algébrique de $u$ et des coëfficients $A_{m p}$. Dans tous les cas on aura:

$$
u=\operatorname{sn}\left(t_{0}+\beta\right)
$$


$t_{0}$ étant la constante d'intégration. On reconnait là le résultat obtenu plus haut.

Arrivons enfin au cas de $p>\mathrm{I}$. Faisons d'abord $z=z_{0}$ dans l'équation (A); cette équation représentera une certaine surface de Ricmarx $S_{0}$. Si l'on y fait $z=z_{1}$, on aura une surface équivalente $S_{1}$. Cette seconde surface dérive de la première par une seule transformation birationnclle ou par un nombre fini de pareilles transformations.

Soit

$$
F_{0}\left(y_{0}, y_{0}^{\prime}\right)=0, \quad\left(\mathrm{~A}_{1}\right) \quad F_{1}\left(y_{1}, y_{1}^{\prime}\right)=0
$$

les équations de ces denx surfuces de Rimass. On passera de l'une à l'autre en posant:

$$
y_{1}=R\left(y_{0}, y_{0}^{\prime}\right), \quad y_{1}^{\prime}=R_{1}\left(y_{0}, y_{0}^{\prime}\right)
$$

$R$ et $R_{1}$ représentant des fonctions rationnelles. Ces fonctions rationnelles $R$ et $R_{1}$ sont telles que l'élimination. de $y_{0}, y_{0}^{\prime}$ entre l'équation $\left(\mathrm{A}_{0}\right)$ et les équations (8) conduit à l'équation $\left(A_{1}\right)$. D'après ce que nous venons de voir, il n'y a qu'un nombre fini de fonctions rationnelles qui jouissent de la même propriété.

Donc quand on connaitra les équations $\left(\mathbf{A}_{0}\right)$ et $\left(\mathbf{A}_{1}\right)$ on pourra trouver les deux fonctions rationnelles $R$ et $R_{1}$ par des procédés purement algébriques.

Comme les coëfficients de $\left(\mathrm{A}_{0}\right)$ et $\left(\mathrm{A}_{1}\right)$ dépendent de $z_{0}$ et de $z_{1}$, il en sera de même des coëfficients de $R$ et $R_{1}$. Si nous regardons $z_{0}$ comme une constante, $z_{1}$ comme la variable indépendante, puis que nous supprimions l'indice I dans $z_{1}, t_{1}, y_{1}, y_{1}^{\prime}$, il viendra, pour l'intégrale générale de $(\mathrm{A})$

$$
y=R\left(y_{0}, y_{0}^{\prime}\right)
$$

$R$ étant une fonction rationnelle des deux constantes d'intégration $y_{0}$ et $y_{0}^{\prime}$, fonction rationnclle dont les coëfficients dépendent de $z$. Les deux constantes d'intégration $y_{0}$ et $y_{0}^{\prime}$ ne sont d'ailleurs pas indépendantes, car elles sont liées entre elles par l'équation $\left(A_{0}\right)$.

Si en particulier l'équation (A) est algébrique en $z$, les coëfficients de $R$ dépendront algébriquement de $z$, et l'intégrale générale de l'équation (A) sera algébrique. 
Si au contraire l'équation (A) s'écrit:

$$
\sum A_{m p} y^{m} y^{p}=\mathrm{o}
$$

les coëfficients $A_{m p}$ étant des fonctions non algébriques en $z$, l'intégrale générale

$$
y=R
$$

sera une fonction algébrique non seulement de $y_{0}$, mais des coëfficients $A_{m p}$.

Dans tous les cas, l'équation (A) s'intègre par des procédés purement algébriques.

On arrive d'ailleurs au même résultat par emploi des fonctions fuchsiennes. Ecrivons encore l'équation (A) sous la forme:

Nous pourrons poser

$$
\sum A_{m p} y^{n} y^{\prime \prime}=\mathrm{o}
$$

$$
y=\varphi(t), \quad y^{\prime}=\varphi(t)
$$

$\varphi$ et $\varphi^{\prime}$ étant deux fonctions fuchsiennes dont les coëfficients dépendent de $z$. Soient $\xi$ et $\eta$ deux fonctions fuchsiennes, indépendantes de $z$ et a. l'aide desquelles toutes les autres sexpriment rationnellement. On aura:

$$
y=R\left(\xi, \gamma_{j}\right), \quad y^{\prime}=R_{1}\left(\xi, \gamma_{j}\right)
$$

$R$ et $R_{1}$ étant des fonctions rationnelles de $\xi$ et de $\eta$. Les coëfficients de ces fonctions rationnelles dépendent de $z$, et il est aisé de voir de quelle manière: ce sont des fonctions algébriques des coëfficients $A_{m p}$.

Conservons aux notations

$$
z_{0}, z_{1}, \quad y_{0}=\varphi\left(t_{0}^{\circ}\right), \quad y_{0}^{\prime}=\varphi^{\prime}\left(t_{0}\right), \quad y_{1}=\varphi\left(t_{1}\right), \quad y_{1}^{\prime}=\varphi\left(t_{1}\right)
$$

le même sens que plus haut. Le point analytique $\left(y_{1}, y_{1}^{\prime}\right)$ devra être lié au point analytique $\left(y_{0}, y_{0}^{\prime}\right)$ par une transformation birationnclle. On aura donc:

la substitution

$$
t_{1}=\frac{\alpha t_{0}+\beta}{i t_{0}+\delta}
$$

$$
\left(t, \frac{a t+\beta}{\gamma t+i}\right)
$$

appartenant au groupe appcé plus haut $G^{\prime}$. 
Les coëfficients $\alpha, \beta, \gamma, \hat{o}$ dépendent de $z_{0}$ et de $z_{1}$; mais ce ne peuvent être que des fonctions continues de $z_{1}$; de plus pour $z_{1}=z_{0}$ on a $t_{1}=t_{0}$. Mais le groupe $G^{\prime}$ est discontinu. Donc on a quels que soient $z_{1}$ et $z_{0}$;

$$
\begin{gathered}
\alpha=\mathrm{i}=\mathrm{1}, \quad \hat{\beta}=\mathrm{O} \\
t_{1}=t_{0} .
\end{gathered}
$$

Ainsi $t$ est une constante et il en est par conséquent de même de $\xi$ et de $\eta$ qui ne dépendent que de $t$. L'intégrale générale de l'équation (A) est donc:

$$
y=R(\xi, y)
$$

oì l'on doit regarder $\xi$ et $\eta$ comme deux constantes d'intégration lices entre elles par une relation algébrique.

C'est le même résultat que plus haut.

Il reste deux questions à résoudre.

On peut se demander en premier lieu s'il existe effectivement des équations intégrables algébriquement par le procédé que nous venons d'indiquer c'est à dire des équations algébriques de la forme (A), de genre $p>\mathrm{I}$ et satisfaisant aux conditions de M. Fucus.

La reponse à cette, première question doit ètre affirmative.

Soit en effet

$$
F(\xi, \eta)=\mathrm{o}
$$

une relation algébrique quelconque de genre $p>$ I. Soit:

$$
x=\varphi(\xi, \eta), \quad y=\phi(\xi, \gamma)
$$

où $\varphi$ et $\psi$ sont des fonctions rationnelles de $\xi$ et de $\eta$ dont les coëfficients dépendent de $z$. Nous supposerons, pour fixer les idées, qu'ils en dépendent algébriquement. L'élimination de $\xi$ et de $\eta$ entre les équations (9) et (10) donnera une relation:

$$
\Phi(x, y, z)=\mathrm{o}
$$

où $\Phi$ est un polynôme entier en $x$ et $y$ dont les coêfficients dépendent de $z$. Considérée comme une relation entre $x$ et $y$ seulement, la relation (I I) définit une surface de Riemans de genre $p$, qui reste équivalente à elle-même quand on fait varier $z$. 
Différentions les relations (IO) par rapport à $z$ (c'est à dire en y regardant $\xi$ et $\eta$ comme des constantes), il viendra:

$$
\frac{d x}{d z}=\frac{d \varphi}{d z}, \quad \frac{d y}{d z}=\frac{d \varphi^{\prime}}{d z} .
$$

$\frac{d \psi}{d z}$ est une fonction rationnelle de $\xi$ et de $\eta$. Maintenant des relations (9) et (I0) on peut déduire les suivantes:

$$
\xi=\varphi_{1}(x, y), \quad \eta=\iota_{1}^{\prime}(x, y)
$$

$\varphi_{1}$ et $\phi_{1}$ étant des fonctions rationnelles de $x$ et de $y$ dont les coëfficients dépendent de $z$. Il viendra donc

$$
\frac{d y}{d z}=R(x, y)
$$

$R$ étant une fonction rationnelle de $x$ et de $y$ dont les coëfficients dépendent de $z$. En éliminant $x$ entre les équations (1 1 ) et (1 3 ) on arrivera à une éqnation de la forme (A), satisfaisant aux conditions de M. Fuchs.

On arriverait an même résultat en éliminant $\xi$ et $\eta$ entre les trois relations:

$$
F(\xi, \eta)=0, \quad y=c^{\prime}(\xi, \eta), \quad \frac{d y}{d z}=\frac{d y^{\prime \prime}}{d z} .
$$

Soit par exemple:

$$
F(u, v, w)=0
$$

une équation dont le premier membre est un polynóme entier homogène du $4^{\mathrm{e}}$ degré en $u, v, w$.

\section{Faisons y}

$$
\begin{aligned}
& u=a x+b y+c \\
& v=a_{1} x+b_{1} y+c_{1} \\
& u=a_{2} x+b_{2} y+c_{2}
\end{aligned}
$$

où les $a$, les $b$ et les $c$ sont des fonctions algébriques de $z$. Nous écrirons l'équation:

$$
F\left(a x+b y+c, a_{1} x+b_{1} y+c_{1}, a_{2} x+b_{2} y+c_{3}\right)=0 .
$$


Nous appellerons $A, A_{1}, A_{2}, B$, etc. les mineurs du déterminant:

Il viendra:

$$
\left|\begin{array}{lll}
a & b & c \\
a_{1} & b_{1} & c_{1} \\
a_{2} & b_{2} & c_{2}
\end{array}\right| .
$$

$$
y=\frac{B u+B_{1} v+B_{2} w}{C u+C_{1} v+C_{2} w}
$$

ou en différentiant par rapport à $z$ et appelant $B^{\prime}, B_{1}^{\prime}$, etc. les dérivées de $B, B_{1}$, etc. par rapport à $z$ :

(I 6$) \frac{d y}{d z}=\frac{\left(B^{\prime} u+B_{1}^{\prime} v+B_{2}^{\prime} w\right)\left(C u+C_{1} v+C_{2} w\right)-\left(C^{\prime} u+C_{1}^{\prime} v+C_{2}^{\prime} w\right)\left(B u+B_{1} v+B_{2} w\right)}{\left(C u+C_{1} v+C_{\mathbf{2}} w\right)^{2}}$.

L'élimination de $u, v, w$ et $x$ entre les équations (14), (15) et (16) donnera une équation différentielle de la forme (A) satisfaisant aux conditions de M. Fuchs.

Passons à la seconde question qu'il nous restait à résoudre:

Etant donnée une équation différentielle algébrique (A), de genre $p>$ I, satisfaisant aux conditions de M. Fucus et que l'on sait par conséquent intégrable algébriquement, comment effectuer réellement cette intégration.

On a vu d'après ce qui précède que cette question se ramène à la suivante: Etant données deux surfaces de Rivaraxx équivalentes, trouver la transformation birationnelle qui permet de passer de l'une à l'autre.

Le problème ainsi posé présente la plus grande analogie avec le problème de la réduction des formes arithmétiques. On conviendra de dire qu'une équation algébrique

$$
F(x, y)=0
$$

est réduite lorsqu'on l'aura ramenée par une transformation birationnęlle à une forme que l'on regardera comme plus simple que toutes les autres. Il faudrait choisir les conditions de réduction de façon:

$I^{\circ}$ que l'on puisse toujours trouver la transformation birationnelle qui réduit une surface de Riemans donnée; 
$2^{\circ}$ qu'il n'y ait en général qu'une seule surface réduite équivalente à une surface de Rresanx donnée et qu'il n'y en ait jamais qu'un nombre fini.

Alors on réduira les deux surfaces $S_{0}$ et $S_{1}$ que l'on veut transformer l'une dans l'autre; si les deux surfaces sont équivalentes, on devra parvenir à la même réduite et, comme on connaîtra la façon de transformer $S_{0}$ en la réduite et la réduite en $S_{1}$, on connaitra aussi la transformation qui change $S_{0}$ en $S_{1}$.

Il est évidemment possible de trouver de pareilles conditions de réduction ce qui rendrait complète l'analogie avec la théorie des formes arithmétiques. Mais cela n'est pas nécessaire; on peut se contenter de conditions de réduction telles que la surface réduite ne dépende plus que d'un nombre fini de paramètres.

Par exemple, Clebsci démontre qu'une courbe de genre $p$

$$
F(x, y)=\text { o }
$$

peut toujours ètre ramenée au degré $p+$ i (ABEL'sche Functionen, p. 65). Après cette réduction, elle dépend encore de $p-\mathrm{I}$ parametres,

Soient donc

$$
F(x, y)=0, \quad F^{\prime}\left(x^{\prime}, y^{\prime}\right)=0
$$

deux courbes de genre $p$ qu'il s'agit de ramener l'une à l'autre par une transformation birationnelle. Je ramènerai la première au degré $p+\mathrm{I}$ par unc transformation convenablement choisie; elle deviendra

$$
F_{1}\left(x_{1}, y_{1}\right)=\mathrm{o}
$$

Je pourrai trouver ensuite, par la méthode de Clebsch une infinité de transformations birationnelles dépendant de $p-\mathrm{I}$ paramètres arbitraires $\alpha_{1}, \alpha_{2}, \ldots, \alpha_{p-1}$, qui ramèneront la seconde courbe au degré $p+1$. Après l'application d'une de ces transformations, l'équation de cette courbe deviendra:

$$
F_{2}\left(x_{1}, y_{1}, \alpha_{1}, \alpha_{2}, \ldots, x_{1,-1}\right)=0
$$

où j'ai mis en évidence les paramètres $\alpha$. Si les deux surfaces de RIEMANN sont équivalentes, on pourra disposer des $\alpha$ de façon à identifier 
les équations (17) et (18) et on connaitra du mème coup la transformation qui fait passer d'une surface à l'autre.

On peut ainsi ramener la recherche des transformations birationnelles qui changent $S_{0}$ en $S_{1}$ à l'étude de l'équivalence des formes algébriques, c'est à dire de la transformation d'une telle forme en une autre par une substitution linéaire.

Soient:

$$
F(x, y)=0, \quad F_{1}\left(x_{1}, y_{1}\right)=0
$$

deux équations qui représenteront deux surfaces de Rremaxx $S_{0}$ et $S_{1}$ et en mème temps deux courbes algebriques $C_{0}$ et $C_{1}$. Soient $m_{0}$ et $m_{1}$ les degrés de ces deux courbes qui auront le mème genre $p$. Soit:

$$
A_{1} \varphi_{1}(x, y)+A_{2} \varphi_{2}(x, y)+\ldots+A_{p} \varphi_{p}(x, y)=\mathrm{o}
$$

l'équation générale des courbes d'ordre $m_{0}-3$ qui passent par tous les points doubles de $C_{0}$. Soit de mème:

$$
B_{1} \psi_{1}\left(x_{1}, y_{1}\right)+B_{2} \psi_{2}\left(x_{1}, y_{1}\right)+\ldots+B_{p} \phi_{p}\left(x_{1}, y_{1}\right)=0
$$

l'équation générale des courbes d'ordre $m_{1}-3$ qui passent par tous les points doubles de $C_{1}$. Soient:

$$
\begin{aligned}
\theta\left(A_{1}, A_{2}, \ldots, A_{p}\right) & =\mathrm{0} \\
\theta_{1}\left(B_{1}, B_{2}, \ldots, B_{p}\right) & =\mathrm{o}
\end{aligned}
$$

les deux relations algébriques et homogènes par rapport aux $A$ et aux $B$ qui expriment, la première que la courbe (19) est tangente à $C_{0}$, la seconde que la courbe (20) est tangente a $C_{1}$. Si d'autre part $x$ et $y$ sont les coordonnées du point de contact des deux courbes (19) et $C_{0}$, si $x_{1}$ et $y_{1}$ sont les coordonnées du point de contact de (20) et de $C_{1}$, on aura:

$$
\begin{array}{ll}
x=R\left(A_{1}, A_{2}, \ldots, A_{p}\right), & y=R^{\prime}\left(A_{1}, A_{2}, \ldots, A_{p}\right) \\
x_{1}=R_{1}\left(B_{1}, B_{2}, \ldots, B_{p}\right), & y_{1}=R_{1}^{\prime}\left(B_{1}, B_{2}, \ldots, B_{p}\right),
\end{array}
$$

les fonctions $R, R^{\prime}, R_{1}$ et $R_{\mathrm{I}}^{\prime}$ étant rationnelles.

Si les deux surfaces de Riemand $S_{0}$ et $S_{1}$ ont mêmes modules, les deux formes algébriques $\theta$ et $\theta_{1}$ seront algébriquement équivalentes, 
c'est à dire qu'on pourra passer de l'une à l'autre par une substitution linéaire, ou en posant:

$$
A_{i}=\sum_{k} a_{i k} B_{k}
$$

les $\alpha_{i k}$ étant des coëfficients constants.

La transformation birationnelle qui change $S_{0}$ en $S_{1}$ sera alors facile a trouver. On aura en effet:

$$
\begin{aligned}
& x=R\left(\sum \alpha_{1 k} B_{1}, \sum \alpha_{2 k} B_{2}, \ldots, \sum \alpha_{p l i} B_{p}\right) \\
& y=R^{\prime}\left(\sum \alpha_{1 k} B_{1}, \sum \alpha_{2 k} B_{2}, \ldots, \sum \alpha_{p k} B_{p}\right)
\end{aligned}
$$

avec les conditions:

(22) $F_{1}\left(x_{1}, y_{1}\right)=0$,

$$
\sum B_{i} \psi_{i}\left(x_{1}, y_{1}\right)=0, \quad \sum B_{i} \theta_{i}\left(x_{1}, y_{1}\right)=0
$$

où l'on a posé:

$$
\theta_{i}=\frac{d \varphi_{i}}{d x_{1}} \frac{d F_{1}}{d y_{1}}-\frac{d \varphi_{i}}{d y_{1}} \frac{d F_{1}^{\prime}}{d x_{1}}
$$

On pourra toujours trouver $p$ fonctions rationnelles de $x_{1}$ et de $y_{1}$ :

$$
\rho_{1}\left(x_{1}, y_{1}\right), \rho_{2}\left(x_{1}, y_{1}\right), \ldots, \rho_{p}\left(x_{1}, y_{1}\right)
$$

qui substituées à la place de $B_{1}, B_{3}, \ldots, B_{p}$ satisfont aux relations (22). On remplacera alors $B_{i}$ par $\rho_{i}\left(x_{1}, y_{1}\right)$ dans les équations $(2 \mathrm{I})$ et on obtiendra ainsi la transformation birationnelle qui change $S_{0}$ en $S_{1}$.

Les invariants qui restent arbitraires dans la forme algébrique $\boldsymbol{\theta}$ sont au nombre de $3 p-3$ et ils doivent être regardés comme les modules de la surface de Riemanis $S_{0}$.

Il est une circonstance sur laquelle je désirerais maintenant attirer l'attention et qui facilite singulièrement soit la recherche des conditions d'équivalence des deux formes $\theta$ et $\theta_{1}$ et de la substitution linéaire qui les transforme l'une dans l'autre.

Parmi les courbes (1 9$)$, il y en a $2^{p-1}\left(2^{p}-\mathrm{I}\right)=P$ qui sont $p-\mathrm{I}$ fois tangentes ̀̀ $C_{0}$; nous les appelerons les courbes $k_{0}$; de même il y aura, parmi les courbes (20), $P$ courbes $k_{1}$ qui seront $p-$ I fois tangentes à $C_{1}$. La substitution linéaire:

$$
A_{i}=\sum \alpha_{i k} B_{k}
$$

qui change $\theta$ en $\theta_{1}$ devra transformer les $P$ courbes $k_{0}$ dans les $P$ 
courbes $k_{1}$. Il pourrait y avoir dans le problème général, une assez grande indétermination; car on pourrait se demander quelle est celle des $P$ courbes $k_{0}$ qui se transformera dans une courbe $k_{1}$ donnée. Il y aurait $\underline{P}$ combinaisons logiquement possibles, ce qui obligerait à faire un nombre très considérable d'essais inutiles.

D'autres considérations viendraient, il est vrai, réduire le nombre des combinaisons logiquement possibles; telle serait par exemple, pour $p=3$, la distribution des 28 tangentes doubles en 64 systèmes de 4 Mais ce nombre n'en resterait pas moins très grand.

Fort heureusement, dans le problème particulier qui nous occupe, cette indétermination n'existe pas. Quelle est celle des courbes $k_{0}$ qui se transforme dans une courbe donnée $k_{1}$ ? La réponse est simple: c'est la courbe $k_{0}$ à laquelle se réduit la courbe donnée $k_{1}$ quand $z_{1}$ se réduit à $z_{0}$.

On arrive même ainsi, presque immédiatement, à déterminer un grand nombre d'intégrales particulières de l'équation (A).

En effet, soit l'équation

$$
F\left(y, y^{\prime}, z\right)=0
$$

pour $z=z_{1}$, elle représente une courbe $C_{1}$, qui est tangente en $P(p-1)$ points aux courbes $k_{1}$. Considérons $y, y^{\prime}$ et $z_{1}$ comme les coordonnées d'un point dans l'espace: lorsqu'on fera varier $z_{1}$, les $P(p-\mathrm{I})$ points de contact $\left(y, y^{\prime}\right)$ de $C_{1}$ avec les $P$ courbes $k_{1}$, décriront dans l'espace $P(p-1)$ courbes qui seront des intégrales particulières de l'équation (A).

Il n'y a donc aucune difficulté à craindre dans les calculs algébriques qui conduisent à l'intégration de l'équation (A).

Remarquons en passant que la considération des $P$ courbes $k_{0}$ nous conduit à une seconde démonstration de ce théorème qu'une surface de Riemann ne peut jamais ètre transformée en elle-même par une infinité de transformations birationnelles.

J'arrive à la conclusion définitive de ce travail. Les équations du ${ }^{\text {er }}$ ordre qui satisfont aux conditions de M. Fucris ne constitucnt pas des classes réellement nouvelles d'équations différentielles.

Dans le cas de $p=0$, elles se ramènent aux équations linéaires.

Dans le cas de $p=1$, elles s'intègrent par une simple quadrature.

Enfin dans le cas de $p>\mathrm{I}$, elles s'intégrent par des procédés purement algébriques. 
Nous devons donc renoncer à l'espoir de rencontrer parmi elles des classes essentiellement nouvelles d'équations intégrables par les transcendantes fuchsiennes. Tout au plus pourrions-nous supposer qu'il en existe de parcilles, parmi les équations d'ordre supérieur; mais on ne pourra s'en assurer que par une discussion spéciale, analogue à celle qui précède.

Le beau résultat de M. Fucis en perd-il pour cela son intérêt? Je ne le crois pas. Il nous fournit en effet une classe très-nombreuse d'équations différentielles intégrables algébriquement. Les conditions de $M$. Fuchs sont très simples et il suffit d'un examen assez rapide pour reconnaitre si elles sont remplies. On reconnait du mème coup l'intégrabilité algébrique, qui sans cette circonstance aurait pu passer inaperçue.

De plus, on peut fonder sur ce théoreme une méthode pour trouver les modules d'une surface de Rismaxx; mais c'est là un point que je ne puis développer en ce moment.

Paris, 25 Novembre 1884 . 